\title{
Severe community-acquired pneumonia: assessment of microbial aetiology as mortality factor
}

\author{
F. Paganin*,\#, F. Lilienthal*, , A. Bourdin*, N. Lugagne ${ }^{+}$, F. Tixier*, R. Génin ${ }^{\S}$, J-L. Yvin ${ }^{\S}$
}

Severe community-acquired pneumonia: assessment of microbial aetiology as mortality factor. F. Paganin, F. Lilienthal, A. Bourdin, N. Lugagne, F. Tixier, R. Génin, J-L. Yvin. (C) ERS Journals Ltd 2004.

ABSTRACT: Community-acquired pneumonia (CAP) remains a major cause of mortality. The aetiology of CAP has rarely been identified as a mortality risk factor. A prospective study was conducted to assess the prognostic factors of CAP patients admitted to the intensive care unit (Centre Hospitalier Departmental Félix Guyon, St Denis de la Réunion, France), with a special emphasis on microbial aetiology.

All variables assessing severity were collected, with a special emphasis on microbial investigations. Among 112 immunocompetent patients (mean \pm SD age $54.7 \pm 15.1$ yrs), $84 \%$ were male. Severity of CAP was demonstrated by mortality rate $(43 \%)$, shock $(48 \%)$, simplified acute physiology score (SAPS; $46.4 \pm 21.6)$ and mechanical ventilation support $(82 \%)$. Mean risk factor score was $2.2 \pm 1.2$. Microbiological identification was obtained in $78.6 \%$ of cases, with positive blood culture in $33 \%$. Most frequently, microbial agents were Streptococcus pneumoniae and Klebsiella pneumoniae $(42 \%$ and $22 \%$, respectively).

The univariate analysis recorded the usual mortality variables: age, alcohol consumption, SAPS, shock, mechanical ventilation, positive end expiratory pressure level, positive blood culture, multilobar infiltrates on chest radiograph, neutropenia, and acidosis, and found $K$. pneumoniae (versus $S$. pneumoniae, and all CAP) as a mortality factor. The multivariate analysis demonstrated that septic shock (relative risk (RR) 141), K. pneumoniae CAP (RR 27), SAPS (RR 10.7) and positive blood culture (RR 2.7) were independent factors related to death.

In conclusion, the present study found that the microbial aetiology, Klebsiella pneumoniae, was an independent risk factor for mortality in severe community-acquired pneumonia.

Eur Respir J 2004; 24: 779-785.
*Service de Réanimation, ${ }^{+}$Service de Bactériologie, and ${ }^{\$}$ Service de Médecine Interne, Centre Hospitalier Departmental Félix Guyon, St Denis de la Réunion, and ${ }^{\#}$ Service de Pneumologie et Maladies Infectieuses, Groupe Hospitalier Sud Reunion (GHSR) St Pierre, Réunion, France. Johnson \& Johnson, Ranitan, NJ, USA.

Correspondence: F. Paganin

Service de Pneumologie et Maladies Infectieuses GHSR, BP 350

St Pierre, Réunion

France

Fax: 262262359128

E-mail: f.paganin@wanadoo.fr

Keywords: Community-acquired pneumonia intensive care unit

Klebsiella pneumoniae

mortality

prognosis

risk factors

Received: October 252003

Accepted after revision: June 172004
Despite advances in the management of severe infectious diseases, community-acquired pneumonia (CAP) remains the major cause of mortality in developed countries [1, 2]. Approximately $10 \%$ of hospitalised patients with CAP require admission to an intensive care unit (ICU), where $20-50 \%$ of them will ultimately die [3, 4].

Many studies have investigated CAP prognosis factors [1-12], and guidelines have been proposed by several medical societies to define the optimal management of patients with CAP [13-17].

The aetiology of CAP is now well known and of worldwide use. However, specific studies suggest a higher frequency of certain pathogens in some geographical areas: Legionella are more common in countries bordering the Mediterranean, Coxiella burnetii in north-west Spain and Canada, Gramnegative bacilli (GNB) in Italy, Burkholderia pseudomallei in South East Asia and Northern Australia, Klebsiella pneumoniae in South Africa, and Mycobacterium tuberculosis in nonindustrialised countries [14]. The knowledge of these particularities is of great importance for physicians dealing in these areas, but also for the medical community, who can be faced everywhere with the diagnosis and prognostic challenges of CAP patients with uncommon pathogens.

In many studies, the rate of microbial identification, even in the ICU, remains extremely low, ranging $10-30 \%$ [2-5, 18-20].
In the majority of published studies, the aetiology of CAP has not been identified as a risk factor (RF) for mortality [2-8, $10,11,21,22]$. Although specific CAP aetiologies, such as $K$. pneumoniae, are frequently suspected of being associated with a higher mortality rate, these aetiologies have not been shown to constitute a prognostic factor for mortality [23-26].

A large prospective study was conducted in order to assess the prognostic factors for mortality of severe CAP patients admitted to the ICU, with a special emphasis on microbial aetiology.

\section{Methods}

\section{Patients}

The 475-bed (16 ICU beds) Centre Hospitalier Departmental Félix Guyon (St Denis de la Réunion, France), where the present study was conducted, is a tertiary institution of a French overseas territory located in the Indian Ocean $2,500 \mathrm{~km}$ east from the South African coast. The island population is estimated to be 750,000 . The medical system and accessibility to medical care is no different to that of France.

The present study prospectively studied 146 consecutive 
patients admitted to the ICU for CAP from September 1995 to December 2000.

All patients had symptoms suggestive of lower respiratory tract infection as generally accepted. Briefly, diagnosis for CAP was a new chest radiological infiltrate and symptoms consistent with pulmonary infection (cough, sputum production, fever $>38.3^{\circ} \mathrm{C}$ or hypothermia, pulmonary consolidation at examination, abnormal leukocytes counts, pleural chest pain). All patients with severe immunosuppression were excluded (known HIV diagnosis, solid organ transplant, current chemotherapy).

Generally, patients were admitted to the emergency room (ER) of the Centre Hospitalier Departmental Félix Guyon. Patients were, secondary, directly admitted to the ICU according to the attending physician's clinical judgement and criteria defining severe CAP $[12,13,27]$. The current authors considered only initial CAP, and excluded progressive pneumonia and early nosocomial pneumonia. All the patients were secondary classified according to the criteria of FINE et al. [28]. When possible, a follow-up visit was performed with clinical parameters analysis, chest radiograph and serological analysis.

\section{Data collection}

In all cases, the following variables were carefully recorded: age, sex, simplified acute physiology score II (SAPS II), prior illness and concomitant conditions, initial signs and symptoms of CAP, alcohol habits, chest radiograph features, arterial blood gases measurements, mechanical ventilation (MV) requirement, and level of positive end expiratory pressure (PEEP). Regarding biological parameters, only initial values were used.

\section{Bacteriological identification procedure}

At least one blood culture was collected for all patients. Serological analysis included the following determination: immunoglobulin (Ig)G versus Mycoplasma pneumoniae, Chlamydia psittaci and $\mathrm{IgG}$, and IgM versus $C$. pneumoniae by indirect immunofluorescence. Legionella pneumophila serotypes 1-6 were diagnosed using the indirect immunofluorescence technique for antibodies detection. ELISA was used to detect $\operatorname{IgM}$ in $M$. pneumoniae. When possible, a second serum sample was obtained on the follow-up visit at 21 days. Urine detection for Legionella antigens was not routinely performed in the ICU.

Pleural fluid culture was performed for patients with significant pleural effusion on chest radiograph. A fibreoptic bronchoscopy with a bronchoalveolar lavage (BAL) was performed, when it was possible, in most patients as a routine procedure in the ICU. No sputum or endotracheal aspiration analysis was performed to obtain bacterial identification.

For BAL, a direct examination (DE) was immediately performed with a cellular differential count (polynuclear, macrophages, lymphocytes, bronchial cells). The absence of bronchial cells or $<5 \%$ was considered as satisfactory for the BAL. For typical pathogens, the authors choose arbitrarily the $\geqslant 1 \times 10^{4} \mathrm{cfu} \cdot \mathrm{mL}^{-1}$ cut-off (recommended for the diagnosis of nosocomial pneumonia) to enhance specificity.

All samples (BAL and pleural fluid) were cultured in adequate media that allowed optimal results (blood agar, chocolate agar, Sabouraud agar, and medium for anaerobes). A microorganism was considered the aetiological agent when blood or pleural fluid cultures were positive and/or if it could be isolated with a cut-off point $\geqslant 1 \times 10^{4} \mathrm{cfu} \cdot \mathrm{mL}^{-1}$ for the BAL. Serologies were considered positive under the following conditions: 1) four-fold increase in IgG titres with final titres for $C$. pneumoniae ( $\mathrm{IgG} \geqslant 512), C$. psittaci $(\mathrm{IgG}$ $\geqslant 64)$, L. pneumophila $(\mathrm{IgG} \geqslant 128)$; 2) increase of IgM titres for $C$. pneumoniae (IgM $\geqslant 32$ ), any positive titre for M. pneumoniae; and 3 ) a single titre $\geqslant 128$ for L. pneumophila.

\section{Definitions}

Shock was defined if initial systolic blood pressure was $\leqslant 80 \mathrm{mmHg}$ after fluid administration or if patients needed vasopressor drug support for $>4 \mathrm{~h}$. Alcohol abuse was considered present when consumption was $\geqslant 120 \mathrm{~g} \cdot \mathrm{day}^{-1}$.

For data obtained in the laboratory, the following criteria were used: hyponatraemia (sodium level $<136 \mathrm{mmol} \cdot \mathrm{L}^{-1}$ ), hyperkaliaemia (potassium level $>5 \mathrm{mmol} \cdot \mathrm{L}^{-1}$ ), acidosis (arterial $\mathrm{pH} \leqslant 7.35$ ), neutropenia (polynuclear cells $\leqslant 1000 \cdot \mathrm{mL}^{-1}$ ), renal failure (creatinine $>200 \mu \mathrm{mol} \cdot \mathrm{L}^{-1}$ and/or blood urea nitrogen $>30 \mathrm{mg} \cdot \mathrm{dL}^{-1}$ ), hypoxaemia at room air (arterial oxygen tension $<60 \mathrm{mmHg}$ ), liver disease (aspartate aminotransferase, alanine aminotransferase $>4$-fold normal value).

Generally, admitted RFs (diabetes mellitus, liver disease, chronic renal failure, cardiac disease, chronic obstructive pulmonary disease (COPD), neurological disease, age $\geqslant 65 \mathrm{yrs}$ ) were recorded in order to obtain a RF score. Each RF was considered as equal.

\section{Statistical analysis}

Prognostic factors were analysed using the Chi-squared test with Fisher's exact test correction, when necessary. For the comparison of means, the Mann-Whitney U-test was used when the variables were not normally distributed; otherwise, the unpaired t-test was used. A p-value $<0.05$ was considered statistically significant. The relative risk for outcome was defined according to the following variables: age ( $>65$ or $<65 \mathrm{yrs})$, alcoholism $\left(>120\right.$ or $\left.<120 \mathrm{~g} \cdot \mathrm{day}^{-1}\right)$, presence of RF (yes/no), number of RF $(\geqslant 2 /<2)$, SAPS $(<40$ or $>40)$, shock (yes/no), MV requirement (yes/no), initial MV (yes/no), level of PEEP $(>10$ or $<10 \mathrm{mmHg}$ ), positive blood culture (yes/no), bilateral involvement on chest radiograph (yes/no), number of lobes involved $(<2$ or $>2)$, microbiological identification (yes/no), type of bacteriological identification (all versus $K$. pneumoniae, all versus Streptococcus. pneumoniae, $S$. pneumoniae versus $K$. pneumoniae), and all of the biological variables, as previously defined.

All of the variables attaining $\alpha$-values $<0.05$ in the univariate analysis were included in the multiple logistic regression analysis model with a stepwise forward selection. All of the first level interactions were tested, excluding all of the variables presenting interaction in the analysis. All reported p-values are two-tailed and the level of significance was set at $5 \%$.

\section{Results}

\section{Patient characteristics}

Among the 146 patients, 34 were excluded as they did not meet a definite diagnosis of CAP ( 25 pulmonary oedema, one pulmonary embolism, and four patients with a normal chest radiograph; five patients had a secondary diagnosis as they were HIV infected with Pneumocystis carinii pneumonia and were subsequently excluded from the analysis). A total of 112 patients $(84 \%$ male, mean age $54.7 \pm 15.1$ yrs) were included in 
the study. The main baseline patient characteristics and underlying conditions are listed in table 1.

Patients were transferred from the ER to the ICU after only $4.0 \pm 2.4 \mathrm{~h}$ (time necessary to confirm the CAP diagnosis and evaluate severity). A total of 42 patients $(62 \%)$ did not receive antibiotics during their ER stay because of rapid orientation to the ICU as a definite diagnosis of CAP was obtained. The present authors did not observe a statistical difference in the delay for antibiotic administration in the patients that were initially treated in the ER compared with those treated in the ICU (3.6 $\pm 3.6 \mathrm{~h}$ versus $3.2 \pm 3.1 \mathrm{~h}$, respectively)).

According to FINE et al. [28], 55 patients in groups 1-3 and 57 in groups 4-5 were found with a statistical difference regarding mortality between these two groups $(\mathrm{p}<0.0045$, relative risk (RR; 95\% confidence interval) 1.92 (1.2-3.09)).

Alcohol abuse was recorded in $62 \%$ of cases and was found to be the most frequent and only RF associated with mortality.

\section{Table 1.-Patient characteristics and underlying conditions}

\begin{tabular}{|c|c|}
\hline Patients & $112(100)$ \\
\hline \multicolumn{2}{|l|}{ Sex } \\
\hline Male & $94(84.0)$ \\
\hline Female & $18(16.0)$ \\
\hline SAPS & $46.4 \pm 21.6$ \\
\hline Risk factors & $2.2 \pm 1.2$ \\
\hline Alcohol abuse & $70(62.5)$ \\
\hline Smoking habit & $49(44.0)$ \\
\hline COPD & $32(28.5)$ \\
\hline Diabetes mellitus & $22(19.6)$ \\
\hline Cardiac disease & $16(14.2)$ \\
\hline Chronic renal failure & $10(8.9)$ \\
\hline Neurologic disease & $7(6.2)$ \\
\hline Immunosuppression & 0 \\
\hline Neoplasia & 0 \\
\hline Temperature at admission ${ }^{\circ} \mathrm{C}$ & $38.0 \pm 1.4$ \\
\hline $\begin{array}{l}\text { Polymorphonuclear neutrophils } \\
\text { cell count }\end{array}$ & $9936 \pm 7781(100-36400)$ \\
\hline Serum $\mathrm{pH}$ & $7.35 \pm 0.15(6.66-7.55)$ \\
\hline \multicolumn{2}{|l|}{$\mathrm{Pa}, \mathrm{O}_{2} \mathrm{mmHg}$} \\
\hline At room air & $53 \pm 10(32-71)$ \\
\hline With nasal $\mathrm{O}_{2} 4 \pm 2 \mathrm{~L}$ & $73 \pm 16(42-120)$ \\
\hline Under $\mathrm{MV}, F \mathrm{I}, \mathrm{O}_{2} 65 \pm 20 \%$ & $96 \pm 46(42-290)$ \\
\hline Overall $\mathrm{Pa}, \mathrm{CO}_{2} \mathrm{mmHg}$ & $38 \pm 13(14-71)$ \\
\hline Serum sodium $\mathrm{mmol} \cdot \mathrm{L}^{-1}$ & $133 \pm 5.4(112-155)$ \\
\hline Serum potassium $\mathrm{mmol} \cdot \mathrm{L}^{-1}$ & $3.2 \pm 1.2(2.4-6.1)$ \\
\hline Serum urea $\mathrm{mmol} \cdot \mathrm{L}^{-1}$ & $7.4 \pm 2.8(2.1-35)$ \\
\hline Serum creatinine $\mu \mathrm{mol} \cdot \mathrm{L}^{-1}$ & $108 \pm 42(52-650)$ \\
\hline Patients with initial MV required & $46(41.1)$ \\
\hline Patients with MV required & $92(82.1)$ \\
\hline Days of MV & $7.1 \pm 7.1$ \\
\hline Shock at admission & $54(48.2)$ \\
\hline \multicolumn{2}{|l|}{ Chest radiograph at admission } \\
\hline Unilateral involvement & $83(74.1)$ \\
\hline Bilateral involvement & $29(25.9)$ \\
\hline Interstitial & $7(6.2)$ \\
\hline Alveolar & $33(29.5)$ \\
\hline Consolidated & $72(64.3)$ \\
\hline Positive blood culture & $37(33.0)$ \\
\hline \multicolumn{2}{|l|}{ Fine's Score (n, \% deceased) } \\
\hline Group 1 & $7(3,47)$ \\
\hline Group 2 & $14(2,14)$ \\
\hline Group 3 & $34(11,32)$ \\
\hline Group 4 & $47(26,55)$ \\
\hline Group 5 & $10(6,60)$ \\
\hline Overall mortality & $48(43)$ \\
\hline
\end{tabular}

Data are presented as $\mathrm{n}(\%)$, mean \pm SD or mean \pm SD (range), unless otherwise stated. SAPS: simplified acute physiology score; COPD: chronic obstructive pulmonary disease; $\mathrm{Pa}, \mathrm{O}_{2}$ : arterial oxygen tension; MV: mechanical ventilation; $\mathrm{FI}, \mathrm{O}_{2}$ : inspiratory oxygen fraction; $\mathrm{Pa}, \mathrm{CO}_{2}$ : carbon dioxide arterial tension.

\section{Radiological and biological findings}

A total of $74 \%$ of patients had a chest radiograph with unilateral involvement. The mean number of lobes involved was $1.8 \pm 0.9$. Consolidated $(64 \%)$ and alveolar (28\%) features were most frequently observed, and were consistent with bacteriological results. No radiological features suggesting a specific aetiology of CAP were observed. Significant pleural effusion was observed in only two cases $(1.7 \%)$. Initial biological values are listed in table 1 .

\section{Bacteriological diagnosis}

Fibreoptic bronchoscopy with BAL was performed in 85 $(76 \%)$ patients and a DE result was obtained in $67(60 \%)$ cases, i.e. presence of Gram-positive cocci, GNB or both.

Microbiological identification was obtained in 88 cases (78.6\%). A positive BAL culture was obtained in $73(65 \%)$ cases. When BAL was negative, a positive blood culture was recorded in $13(11.6 \%)$ cases and significant positive serology in two cases. BAL and blood cultures were both positive in 24 $(21 \%)$ of the cases. No pleural effusion analysis was positive.

The most frequently isolated aetiological agents were $S$. pneumoniae and $K$. pneumoniae $(42 \%$ and $22 \%$ of patients, respectively). Other microbial agents (Escherichia coli, Moraxella catarrhalis, Staphylococcus aureus, Pseudomonas aeruginosa, L. pneumophila, $H$. influenzae, Morganella morgani) were found in $<3 \%$ of cases, and are listed in table 2 . Only three cases $(2.6 \%)$ were found of $S$. pneumoniae CAP associated with Haemophilus influenzae. Due to predominant pathogen, these cases of CAP were considered as $S$. pneumoniae CAP. One case of aspergillosis was recorded by a positive BAL and corresponded to subacute necrotising pneumonia.

Overall, a positive blood culture was obtained in $37(33 \%)$ of patients, and in $48 \%$ and $36 \%$ of patients with $S$. pneumoniae CAP and $K$. pneumoniae CAP, respectively. MV support was required for $88 \%$ of $K$. pneumoniae patients and $76 \%$ of $S$. pneumoniae patients (nonsignificant).

The high rate of klebsiella pathogens is strongly related to alcoholism. A significant specific distribution of CAP related to $K$. pneumoniae was found in the group of alcoholic patients compared to nonalcoholic patients ( $88 \%$ versus $12 \%$, RR (95\% confidence interval) $4.5(1.45-14.35), \mathrm{p}<0.002)$. However, this finding is not very discriminatory, as a high percentage of alcoholic patients with a $S$. pneumoniae CAP

Table 2.-Microbial identification

\begin{tabular}{lccc}
\hline Aetiology & & $\begin{array}{c}\text { Positive blood } \\
\text { culture }\end{array}$ & $\begin{array}{c}\text { Mortality } \\
\text { rate }\end{array}$ \\
\hline Identified & $88(78.6)$ & $37(33)$ & $41(36.7)$ \\
$\begin{array}{l}\text { Streptococcus pneumoniae } \\
\quad 48(42.9)\end{array}$ & $23(48)$ & $17(35.4)$ \\
Positive DE on BAL & $39(81)$ & & \\
Klebsiella pneumoniae & $25(22.4)$ & $9(36)$ & $18(72)$ \\
$\quad$ Positive DE on BAL & $21(84)$ & & \\
Escherichia coli & $3(2.6)$ & $1(33.3)$ & $2(66.7)$ \\
Moraxella catarrhalis & $3(1.8)$ & 0 & 0 \\
Staphylococcus aureus & $2(1.8)$ & $1(50)$ & $1(50)$ \\
Pseudomonas aeruginosa & $2(1.8)$ & $2(100)$ & $2(100)$ \\
Legionella pneumophilia & $2(1.8)$ & $\mathrm{NA}$ & 0 \\
Haemophilus influenzae & $1(0.9)$ & 0 & 0 \\
Morganella morganii & $1(0.9)$ & $1(100)$ & 0 \\
Aspergillus niger & $1(0.9)$ & $\mathrm{NA}$ & $1(100)$ \\
None identified & $24(21.4)$ & 0 & $7(29)$ \\
\hline Dota & & &
\end{tabular}

Data are presented as $\mathrm{n}(\%)$. DE: direct examination; BAL: bronchoalveolar lavage fluid; NA: not available. 
(64\%) was also found, but with no significant statistical correlation.

\section{Antibiotic regimens for treatment of pneumonia}

All patients were treated with adequate i.v. antibiotics depending on the physician's judgment. All patients received at least a third-generation cephalosporin in 70 cases $(62.5 \%)$, or amoxicillin/clavulanic acid in 42 cases $(37.5 \%)$. Monotherapy was administrated in 43 cases (38\%) as follows: thirdgeneration cephalosporin in $33(77 \%)$ and amoxicillin/clavulanic acid in $10(23 \%)$. Aminoglycosides were added in 33 cases $(29.5 \%)$, macrolide or quinolone (ofloxacin) in 29 cases $(26 \%)$ and multiple regimens ( $\beta$-lactam+aminoglycoside+quinolone) in seven cases $(6 \%)$. Addition of antibiotics was guided by microbiological results (i.e. DE), and occurred within the first $24 \mathrm{~h}$ after admission.

A total of $42(37.5 \%)$ patients received antibiotics before admission in the ICU (initial antibiotics). Among the 15 patients that were treated by their general practitioner before ER admission, antibiotic regimen was continued in seven cases (i.v. third-generation cephalosporin) and modified in eight cases (i.v. antibiotic administration: amoxicillin/clavulanic acid in six cases, and i.v. third-generation cephalosporin in two cases, in substitution of macrolides in seven cases and cyclines in one case). The $70(62.5 \%)$ other patients received antibiotics at admission in the ICU. Among patients treated with antibiotics in the ER, it was possible to obtain a microbial identification in 20 cases $(47 \%)$, as it was obtained in $68(97 \%)$ of the 70 remaining patients that received antibiotics in ICU. The results of bacteriological procedures (positive DE or positive BAL), and the administration of antibiotics before admission in ICU did not influence mortality (table 3 ). The authors did not observe differences in outcome for patients that received monotherapy versus multiple antibiotic regimens ( $R R 0.76, p=0.33$ ). However, the decision of administrating antibiotic treatment was different for patients that received antibiotics in the ER (monotherapy in $8 \%$ ) compared with the group that received antibiotics in the ICU (monotherapy in $31 \% ; \mathrm{p}<0.008$ ).

The resistance of $S$. pneumoniae to penicillin was observed in only two cases $(4 \%)$, with one case of intermediate resistance and one case of high-level resistance. All of the $K$. pneumoniae had an in vitro sensitivity to third-generation cephalosporin, amoxicillin/clavulanic acid, aminoglycosides and quinolones. None of the strains expressed extendedspectrum $\beta$-lactamases.

\section{Prognostic factors}

The severity of the CAP diagnosed in this study was demonstrated by a high mortality rate (43\%), a high percentage of patients with initial shock (48\%), and initial MV support at admission (41\%). All the patients, except two, who were discharged from the ICU survived. The univariate analysis recorded variables related to death and are listed in table 4.

Table 5 shows the results of the multivariate analysis of prognosis factors. The logistic regression demonstrated that septic shock (RR 141), CAP with a Klebsiella aetiology (RR 27), SAPS II score $>40$ (RR 10.7) and positive blood culture (RR 2.7) were the only independent factors related to death.

Table 6 presents clinical, radiological and biological data on Klebsiella CAP compared with CAP of other aetiology.

\section{Discussion}

A large prospective study of nonimmunocompromised patients, presenting with severe CAP, is herein reported. A special emphasis was given to the microbiological identification, in order to assess the microbial aetiology as a potential prognostic factor for mortality.

Two important findings were drawn from this study. $K$. pneumoniae was identified as the causative pathogen in $22 \%$ of cases, second to $S$. pneumoniae. Moreover, $K$. pneumoniae was identified as an independent mortality factor for CAP.

The study population was characterised by a large percentage of males. Unlike most severe CAP studies, classical RFs, such as COPD, renal failure, heart diseases and diabetes mellitus, were present in only a minority of patients $[2,4,6,9,29]$. Alcohol abuse was the only RF correlated with death, but was also found to be more frequent in $K$. pneumoniae CAP compared with other CAPs (table 6). In the literature, alcoholism is generally associated with severe CAP and, particularly, with $K$. pneumoniae CAP $[30,31]$. However, the current authors found about half of patients with alcohol abuse in the non-K. pneumoniae group. Alcoholism was correlated with death (table 3), but did not appear to be an independant mortality factor in the multivariate analysis, as alcoholism was widely distributed in the studied patients. Alcohol abuse cannot be a factor, suggesting a specific microbial aetiology for CAP. The relatively young age and the low representation of RFs were in contrast with the high severity of the population that was reflected by a high rate of required $\mathrm{MV}$ and septic shock, a high-severity SAPS score and, finally, a high crude mortality rate of $43 \%$.

Radiological features were consistent with the microbiological epidemiology. A large proportion of unilateral, alveolar and consolidated infiltrates reflected the high incidence of $S$. pneumoniae and $K$. pneumoniae. Bilateral involvement was found in $26 \%$ of cases, with half of them caused by $S$. pneumoniae and $K$. pneumoniae. Bilateral infiltrates appeared to result from the rapid extension of a typical pneumonia. Of the 24 CAP without a microbial diagnosis, 17 had a unilateral infiltrate, suggesting a pathogen related to a

Table 3. - Influence of antibiotic therapy and identification technique

\begin{tabular}{|c|c|c|c|c|c|}
\hline & Survivors ${ }^{\#}$ & Nonsurvivors & Relative risk & $95 \% \mathrm{CI}$ & $\mathrm{p}$-value \\
\hline \multicolumn{6}{|l|}{ Initial $\mathrm{AB}$} \\
\hline Administered by GP & $9(14)$ & $6(12.5)$ & 1.0 & $0.67-1.65$ & $1.00 \mathrm{NS}$ \\
\hline Administered in ER & $23(20.5)$ & $19(17)$ & 1.06 & $0.65-1.71$ & $0.84 \mathrm{NS}$ \\
\hline $\mathrm{AB}$ regimen (use of monotherapy) & $27(24)$ & $16(14)$ & 0.76 & $0.46-1.24$ & $0.33 \mathrm{NS}$ \\
\hline Monotherapy (for S. pneumoniae) & $17(26.5)$ & $8(16)$ & 0.83 & $0.45-1.5$ & $0.55 \mathrm{NS}$ \\
\hline Examination of BAL (positive DE) & $34(30)$ & $33(29.5)$ & 1.28 & $0.94-1.74$ & $0.12 \mathrm{NS}$ \\
\hline Positive culture of BAL & $37(33)$ & $36(32)$ & 1.20 & $0.92-1.57$ & $0.23 \mathrm{NS}$ \\
\hline
\end{tabular}

Data are presented as n (\%), unless otherwise stated. CI: confidence interval; AB: antibiotics; GP: general practitioner; ER: emergency room; $S$. pneumoniae: Streptococcus pneumoniae; BAL: bronchoalveolar lavage fluid; DE: direct examination. NS: nonsignificant. ${ }^{\#}: \mathrm{n}=64$; ${ }^{\natural}: \mathrm{n}=48$. 
Table 4.- Univariate analysis of prognostic factors

\begin{tabular}{|c|c|c|c|c|c|}
\hline & Survivors ${ }^{\#}$ & Nonsurvivors & Relative risk & $95 \% \mathrm{CI}$ & p-value \\
\hline Age $>65$ yrs & 0 & $8(7)$ & 2.6 & $2.03-3.31$ & 0.0008 \\
\hline Alcoholism & $29(26)$ & $40(36)$ & 3.11 & $1.61-6.00$ & 0.0001 \\
\hline Presence of RF & $27(51)$ & $46(41)$ & 2 & $0.58-6.95$ & $0.29 \mathrm{NS}$ \\
\hline Number of $\mathrm{RF}>2$ & $44(39)$ & $34(30)$ & 0.93 & $0.5-1.6$ & $0.83 \mathrm{NS}$ \\
\hline Fine's score $1-3 / 4-5$ & $39 / 25$ & $16 / 32$ & 1.92 & $1.2-3.09$ & 0.0045 \\
\hline SAPS II score $>40$ & $16(14)$ & $40(36)$ & 2.93 & $1.91-4.51$ & 0.0001 \\
\hline Septic shock & $9(8)$ & $43(39)$ & 12.08 & $46-31.4$ & 0.0001 \\
\hline MV & $42(38)$ & 49 (44) & 11.3 & $1.65-77.3$ & 0.0001 \\
\hline Initial MV & $23(21)$ & $23(21)$ & 1.26 & $0.83-1.92$ & $0.33 \mathrm{NS}$ \\
\hline PEEP $>10 \mathrm{mmHg}$ & $7(8)$ & $25(28)$ & 2.18 & $1.47-3.25$ & 0.0002 \\
\hline Positive blood culture & $15(13)$ & $23(21)$ & 1.72 & $1.15-2.57$ & 0.015 \\
\hline \multicolumn{6}{|l|}{ Initial chest radiograph } \\
\hline Bilateral infiltrate & $18(16)$ & $11(10)$ & 0.80 & $0.48-1.35$ & $0.5 \mathrm{NS}$ \\
\hline Multilobar infiltrate $^{+}$ & $8(7)$ & $15(13)$ & 1.7 & $1.14-2.54$ & 0.03 \\
\hline Neutropenia $<1000$ cells $\cdot \mathrm{mm}^{3}$ & $5(4)$ & $20(18)$ & 2.48 & $1.72-3.57$ & 0.0001 \\
\hline Acidosis $\mathrm{pH}<7.35 \mathrm{mmol} \cdot \mathrm{L}^{-1}$ & $13(12)$ & $36(32)$ & 2.20 & $1.49-3.24$ & 0.0001 \\
\hline Sodium level $<136 \mathrm{mmol} \cdot \mathrm{L}^{-1}$ & $28(25)$ & $30(27)$ & 0.9 & $0.67-1.36$ & $0.8 \mathrm{NS}$ \\
\hline Potassium level $>5 \mathrm{mmol} \cdot \mathrm{L}^{-1}$ & $6(5)$ & $12(11)$ & 1.56 & $1.04-2.34$ & $0.07 \mathrm{NS}$ \\
\hline Renal failure & $8(7)$ & $2(1.8)$ & 0.32 & $0.07-1.44$ & $0.18 \mathrm{NS}$ \\
\hline Hypoxaemia & $17(15)$ & $21(19)$ & 1.51 & $1.0-2.29$ & $0.07 \mathrm{NS}$ \\
\hline No microbial identification & $16(14)$ & $8(7)$ & 0.71 & $0.38-1.31$ & $0.3 \mathrm{NS}$ \\
\hline \multicolumn{6}{|l|}{ Aetiology of CAP } \\
\hline S. pneumoniae versus all & $30(27)$ & $17(15)$ & 0.75 & $0.48-1.19$ & $0.2 \mathrm{NS}$ \\
\hline Klebsiella versus all & $7(6)$ & $18(16)$ & 2.02 & $1.39-2.93$ & 0.0025 \\
\hline Klebsiella versus $S$. pneumoniae & $7(10)$ & $18(25)$ & 1.99 & $1.26-3.12$ & 0.006 \\
\hline
\end{tabular}

Data are presented as $\mathrm{n}(\%)$, unless otherwise stated. Percentages in parentheses indicate values calculated from the total of patients, except the positive end expiratory pressure (PEEP, $\mathrm{n}=88$ ) and aetiology Klebsiella versus Streptococcus pneumoniae $(S$. pneumoniae, $\mathrm{n}=72)$. CI: confidence interval; RF: risk factor; SAPS: simplified acute physiology score; MV: mechanical ventilation; CAP: community-acquired pneumonia. NS: nonsignificant. ${ }^{\#}: \mathrm{n}=64 ;{ }^{\top}: \mathrm{n}=48 ;{ }^{+}:>2$ lobes.

Table 5. - Multivariate analysis of prognostic factors

\begin{tabular}{lcccr}
\hline & Survivors $^{\#}$ & Nonsurvivors & Relative risk & p5\% CI \\
\hline Septic shock & $9(8)$ & $43(39)$ & 141 & 0.0001 \\
Klebsiella versus all & $7(6)$ & $18(16)$ & 27 & 0.0001 \\
SAPS II score $>40$ & $16(14)$ & $40(36)$ & 10.7 & $25-1494$ \\
Positive blood culture & $15(13)$ & $23(21)$ & 2.7 & $3.1-3.7$ \\
\hline
\end{tabular}

Data are presented as $\mathrm{n}(\%)$, unless otherwise stated. CI: confidence interval; SAPS: simplified acute physiology score. ${ }^{*}$ : $\mathrm{n}=64 ;{ }^{\top}: \mathrm{n}=48$.

Table 6. - Characteristics of Klebsiella community-acquired pneumonia (CAP) patients compared to CAP of other aetiology

\begin{tabular}{lccc}
\hline & $\begin{array}{c}\text { Klebsiella } \text { CAP }^{\#} \\
\text { CAP }\end{array}$ & $\begin{array}{c}\text { Other } \\
\text { C-value }\end{array}$ \\
\hline Male:female & $24: 1$ & $75: 12$ & NS \\
Age yrs & $56 \pm 13$ & $54 \pm 15$ & NS \\
Alcohol abuse & $21(84)$ & $49(56)$ & $<0.001$ \\
Temperature at admission ${ }^{\circ} \mathrm{C}$ & $37.5 \pm 1.3$ & $38.1 \pm 1.4$ & $\mathrm{NS}$ \\
Polymorphonuclear cells & $8412 \pm 7294$ & $10357 \pm 7898$ & $\mathrm{NS}$ \\
Chest radiograph & & & \\
$\quad$ Number of lobes involved & $1.7 \pm 0.8$ & $1.7 \pm 0.9$ & \\
$\quad$ Unilateral involvement & $19(76)$ & $64(82)$ & \\
$\quad$ Bilateral involvement & $6(24)$ & $23(26)$ & NS \\
$\quad$ Interstitial & 0 & $7(8)$ & \\
$\quad$ Alveolar & $5(20)$ & $28(32)$ & \\
$\quad$ Consolidated & $14(56)$ & $58(66)$ & \\
SAPS II score & $55.3 \pm 26.9$ & $43.9 \pm 19$ & NS \\
Shock & $15(60)$ & $39(45)$ & NS \\
Mortality & $18(72)$ & $30(34)$ & $<0.002$ \\
\hline
\end{tabular}

Data are presented as $\mathrm{n}, \mathrm{n}(\%)$ or mean \pm SD. SAPS: simplified acute physiology score. NS: nonsignificant. ${ }^{\#}: n=25 ;{ }^{\uparrow}: n=87$. typical CAP. Some radiological features were attributed to K. pneumoniae. Radiographical description for the Friedlander pneumoniae focused on the bulging fissure sign, highly suggesting $K$. pneumoniae species when present. Unfortunately, this fissure sign was not found, except in one case. The present authors, at least, did not find any radiographical features that could predict a specific aetiology (table 6).

A microbial diagnosis was established in $78.6 \%$ of patients due to the frequent utilisation of fibreoptic techniques routinely used by chest physicians and trained anaesthesiologists in the ICU. At least, microbial identification was more frequent in patients that did not receive antibiotics before admission into the ICU. BAL was performed in $85(76 \%)$ patients, along with blood cultures, and serologies were systematically performed in all patients. No complications were experienced in any patients that underwent BAL. S. pneumoniae was isolated in $43 \%$ of patients. This result is consistent with those reported in severe [2-11, 18, 19-23] and nonsevere CAP $[12,32,33]$. However, the high frequency of isolated $K$. pneumoniae $(22 \%)$ is in contrast with the low percentage reported in most published CAP series, with the exception of studies conducted by FELDMAN et al. [30, 31] in South Africa. The current authors have no hypothesis to 
explain these similarities, except the location in the south hemisphere region, $2,500 \mathrm{~km}$ east of South Africa. The type of alcohol abuse is specific on Reunion Island (high-proof agricol rum), but no data was found to support a relation with high $K$. pneumoniae incidence. Alcoholism and poor underlying conditions are known predisposing factors of CAP caused by either $K$. pneumoniae or $S$. pneumoniae, and are frequently reported in similar proportion in published studies $[6,34]$.

Surprisingly, only two patients were diagnosed with an atypical pathogen. Both cases were caused by Legionella and were imported from Europe. The diagnosis of atypical pathogens may have been underestimated, as a second serology could not be obtained in most patients who died within the first 2 weeks after admission. However, the knowledge of the local epidemiology does not support the fact that Legionella is a common pathogen in Réunion, France.

A univariate analysis identified several prognostic RFs for mortality: age, alcoholism, SAPS II (>40), septic shock, mechanical ventilation, PEEP $(>1.33 \mathrm{kPa}(>10 \mathrm{mmHg}))$, multilobar infiltrate, positive blood cultures, neutropenia and acidosis. A multivariate analysis of these factors only found septic shock, SAPS II and positive blood cultures to be independently associated with mortality. All these findings were consistent with those reported in the literature [2-12].

The major objective of this study was to assess whether the microbiological aetiology could be a factor associated with fatal outcome. $K$. pneumoniae was shown to be a prognostic factor for mortality in a univariate analysis, but also in the multivariate analysis (RR 27). In the literature, GNB were identified as a mortality factor for CAP [9]. However, among 14 cases of GNB, they found six cases with $K$. pneumoniae and seven cases with $E$. coli, with a mortality of $33 \%$ and $43 \%$, respectively. A high percentage $(72 \%)$ of microbiological aetiology could explain these results. In the present study, the rate and the mortality of $K$. pneumoniae was extremely high $(72 \%)$, and this subgroup was not matched with other GNB. It is important to notice that $S$. pneumoniae was not found to be associated with fatal outcome in the univariate analysis, whether with a positive blood culture or not. In the current study, the results of MoINE et al. [9] could not be confirmed that found $S$. pneumoniae associated with death. It is noteworthy to point out that the crude mortality of $S$. pneumoniae was equal in the study by MoINE et al. [9] and the present study $(35 \%)$. Some authors have focused on the potential severity of some bacteriological species (Pseudomonas aeruginosa) in nosocomial pneumonia $[35,36]$, or in CAP $[6,37]$ when structural lung diseases are present (bronchiectasis) [38]. In CAP, the pathogens generally implicated in severe cases have failed to demonstrate a role in poor outcome.

The potential influence of antibiotic regimen is important for the outcome [39]. The choice of antibiotic regimen administrated at admission in the ICU was effective regarding the bacterial results recorded throughout the study. A total of $15(13 \%)$ patients received antibiotics by their general physician before admission to the hospital. Most patients $(62 \%)$ did not receive any antibiotic treatment during their brief ER stay and rapidly underwent microbiological procedures upon their ICU admission. No differences were seen in the outcome for patients that received antibiotics in the ER and those that received antibiotics after admission in the ICU. For the group of patients that were not given antibiotic, the mean time from admission to diagnosis in the ICU was $3.2 \pm 3.1 \mathrm{~h}$, compared with the pretreated group in the ER $(3.6 \pm 3.6 \mathrm{~h})$. This result supports the fact that a direct admission in the ICU without antibiotic therapy did not influence patient outcome, but improved the possibility to obtain a microbial identification. A rapid result with a positive DE of the BAL was helpful for antibiotic therapy decision, as recently pointed out [37]. GNB observed on the BAL DE highly suggested a $K$. pneumoniae CAP, as Gram-positive cocci suggested $S$. pneumoniae CAP. Guided by a positive DE of the BAL, monotherapy (third-generation cephalosporin or amoxicillin/clavulanic acid) was administrated in $25(52 \%)$ of patients with $S$. pneumoniae. This decision did not influence outcome, as shown in table 3. The knowledge of high antibiotic sensitivity of the strains of $S$. pneumoniae recorded in the area, and the chest radiograph presentation (inconsistent with atypical pathogens), supported the choice of monotherapy in some patients of the $S$. pneumoniae group. The use of two antibiotics ( $\beta$-lactam + macrolide or quinolone) is generally indicated to extend the antibiotic spectrum against atypical pathogens [13-15]. Moreover, the addition of an aminoglycoside can be recommended in case of severe CAP [13-15]. The addition of an aminoglycoside in some CAP patients of the $S$. pneumoniae group and in other patients with non-S. pneumoniae aetiologies did not modify survival. In all cases of GNB, observed at DE of the BAL, an aminoglycoside was immediately administrated in association with a $\beta$-lactam antibiotic. According to this antibiotic strategy and the overall bacterial sensitivity, the poor outcome in CAP patients in this study cannot be explained by the antibiotic treatment [40].

In summary, this large prospective study showed that Klebsiella pneumoniae was not only a frequent aetiology of severe community-acquired pneumonia patients, but also an independent risk factor for mortality in this population. The high rate of patients with a microbial diagnosis $(\sim 80 \%)$ significantly contributed towards demonstrating these findings, but a contrario represented a limit of the study, as bronchoscopic procedures are not routinely performed everywhere. As Klebsiella pneumoniae is certainly more frequent in Réunion than in other countries, this aetiology can be found worldwide in patients with some underlying conditions, such as alcohol abuse. However, the authors failed to find some clinical and radiological particularities, suggesting this aetiology in patients at admission. Further studies on the epidemiology and prognostic risk factors of severe community-acquired pneumonia are warranted to assess if early recognition of microbial aetiology can modify the outcome of these severe community-acquired pneumonias.

Acknowledgements. The authors would like to thank J.P. Daures for his kind help in the statistical analysis. This work is dedicated to the family of F. Paganin.

\section{References}

1. Marrie TH, Durant H, Yates L. Community-acquired pneumonia requiring hospitalization: 5-year prospective study. Rev Infect Dis 1989; 11: 86-99.

2. Torres A, Serra-Batlles J, Ferrer A, et al. Severe communityacquired pneumonia. Epidemiology and prognostic factors. Am Rev Respir Dis 1991; 144: 312-318.

3. Rello J, Quintana E, Aussina V, Net A, Prats G. A threeyear study of severe community-acquired pneumonia with emphasis on outcome. Chest 1993; 103: 232-235.

4. Almirall J, Mesalles E, Klamburg J, Parra O, Agudo A. Prognostic factors of pneumonia requiring admission to the intensive care unit. Chest 1995; 107: 511-516.

5. Woodhead MA, MacFarlane JT, Rodgers FG, Laverick A, Pilkington R, Macrae AD. Aetiology and outcome of severe community-acquired pneumonia. J Infect 1985; 10: 204-210. 
6. Ruiz M, Ewig S, Marcos MA, et al. Etiology of communityacquired pneumonia: impact of age, comorbidity, and severity. Am J Respir Crit Care Med 1999; 160: 397-405.

7. Ruiz M, Ewig S, Torres A, et al. Severe community-acquired pneumonia: risk factors and follow-up epidemiology. $\mathrm{Am}$ J Respir Crit Care Med 1999; 160: 923-929.

8. Ewig S, Ruiz M, Mensa J, et al. Severe community-acquired pneumonia. Assessment of severity criteria. Am J Respir Crit Care Med 1998; 158: 1102-1108.

9. Moine P, Vercken JB, Chevret S, Chastang C, Gajdos P. Severe community-acquired pneumonia. Etiology, epidemiology, and prognosis factors. French Study Group for Community-Acquired Pneumonia in the Intensive Care Unit. Chest 1994; 105: 1487-1495.

10. Georges H, Leroy O, Vandenbussche C, et al. Epidemiological features and prognosis of severe communityacquired pneumococcal pneumonia. Intensive Care Med 1999; 25: 198-206.

11. Ewig S, Schafer H, Torres A. Severity assessment in community-acquired pneumonia. Eur Respir J 2000; 16: 1193-1201.

12. Fine MJ, Smith MA, Carson CA, et al. Prognosis and outcomes of patients with community-acquired pneumonia. A meta-analysis. JAMA 1996; 275: 134-141.

13. Niederman MS, Mandell LA, Anzueto A, et al. Guidelines for the management of adults with community-acquired pneumonia. Diagnosis, assessment of severity, antimicrobial therapy, and prevention. Am J Respir Crit Care Med 2001; 163: 1730-1754.

14. British Thoracic Society Standards of Care Committee. BTS guidelines for the management of community-acquired pneumonia in adults. Thorax 2001; 56: Suppl. 4, 1-64.

15. Niederman MS. Severe community-acquired pneumonia: what do we need to know to effectively manage patients? Intensive Care Med 1996; 22: 1285-1287.

16. Hirani NA, Macfarlane JT. Impact of management guidelines on the outcome of severe community acquired pneumonia. Thorax 1997; 52: 17-21.

17. Mandell LA, Marrie TJ, Grossman RF, Chow AW, Hyland RH. Canadian guidelines for the initial management of community-acquired pneumonia: an evidence-based update by the Canadian Infectious Diseases Society and the Canadian Thoracic Society. The Canadian CommunityAcquired Pneumonia Working Group. Clin Infect Dis 2000; 3: 383-421.

18. Pachon J, Prados MD, Capote F, Cuello JA, Garmacho J, Verano A. Severe community-acquired pneumonia: aetiology, prognosis, and treatment. Am Rev Respir Dis 1990; 142: 369-373.

19. Ortqvist A, Sterner G, Nilsson JA. Severe communityacquired pneumonia: factors influencing need of intensive care treatment and prognosis. Scand J Infect Dis 1985; 17: 377-386.

20. Marrie TH, Peeling RW, Fine MJ, Singer DE, Coley CM, Kapoor WM. Ambulatory patients with communityacquired pneumonia: the frequency of atypical agents and clinical course. Am J Med 1996; 101: 508-515.

21. el-Ebiary M, Sarmiento X, Torres A, et al. Prognostic factors of severe Legionella pneumonia requiring admission to ICU. Am J Respir Crit Care Med 1997; 156: 1467-1472.

22. Torres A, el-Ebiary M. Prognosis factors in severe communityacquired pneumonia: a step forward. Intensive Care Med 1996; 22: 1288-1290.
23. Leroy O, Santre C, Beuscart C, et al. A five-year study of severe community-acquired pneumonia with emphasis on prognosis in patients admitted to an intensive care unit. Intensive Care Med 1995; 21: 24-31.

24. Marrie TJ. Community-acquired pneumonia. Clin infect Dis 1994; 18: 501-513.

25. Bartlett JG, Mundy LM. Current concepts: communityacquired pneumonia. N Engl J Med 1995; 333: 1618-1624.

26. Feldman C, Ross S, Mahomed AG, Omar J, Smith C. The aetiology of severe community-acquired pneumonia and its impact on initial, empiric, antimicrobial chemotherapy. Respir Med 1995; 89: 187-192.

27. American Thoracic Society. Guidelines for the initial management of adults with community-acquired pneumonia: diagnosis, assessment of severity, and initial antimicrobial therapy. Am Rev Respir Dis 1993; 148: 1418-1426.

28. Fine MJ, Auble TE, Yealy DM, et al. A prediction rule to identify low-risk patients with community-acquired pneumonia. N Engl J Med 1997; 336: 243-250.

29. Rello J, Rodriguez R, Jubert P, Alvarez B. Severe community-acquired pneumonia in the elderly: epidemiology and prognosis. Study Group for Severe Community-Acquired Pneumonia. Clin Infect Dis 1996; 23: 723-728.

30. Feldman C, Kallenbach JM, Levy H, Thorburn JR, Hurwitz $\mathrm{MD}$, Koornhof HJ. Comparison of bacteraemic communityacquired lobar pneumonia due to Streptococcus pneumoniae and Klebsiella pneumoniae in an intensive care unit. Respiration 1991; 58: 265-270.

31. Feldman C, Kallenbach JM, Levy H, et al. Communityacquired pneumonia of diverse aetiology: prognostic features in patients admitted to an intensive care unit and a "severity of illness" core. Intensive Care Med 1989; 15: 302-307.

32. Woodhead MA, Macfarlane JT, McCracken JS, Rose DH, Finch RG. Prospective study of the aetiology and outcome of pneumonia in the community. Lancet 1987; 1: 671-674.

33. Berntsson E, Lagergard T, Stannegard O, Trollfors B. Etiology of community-acquired pneumonia in outpatients. Eur J Clin Microbiol 1986; 5: 446- 447.

34. Perlino CA, Rimland D. Alcoholism, leukopenia and pneumococcal sepsis. Am Rev Respir Dis 1985; 132: 757760.

35. Fagon JY, Chastre J, Domard Y, et al. Nosocomial pneumonia in patients receiving continuous mechanical ventilation: prospective analysis of 52 episodes with use of a protected specimen brush and quantitative techniques. $\mathrm{Am}$ Rev Respir Dis 1989; 139: 877-884.

36. Dunn M, Wunderink RG. Ventilator associated pneumonia caused by pseudomonas infection. Clin Chest Med 1995; 16 : 95-109.

37. Rello J, Bodi M, Mariscal D, et al. Microbial testing and outcome of patients with severe community-acquired pneumonia. Chest 2003; 124: 1179-1180.

38. El-Solh AA, Sikka P, Ramadan F, Davies J. Etiology of severe pneumonia in the very elderly. Am J Respir Crit Care Med 2001; 163: 645-651.

39. Waterer GW, Jennings SG, Wunderink RG. The impact of blood cultures on antibiotic therapy in pneumococcal pneumonia. Chest 1999; 116: 1353-1355.

40. Valles J, Rello J, Ochagavia J, Alcala MA. Communityacquired bloodstream infection in critically ill adult patients: impact of shock and inappropriate antibiotic therapy on survival. Chest 2003; 123: 1615-1624. 